\title{
LOS NIDOS ARTIFICIALES ASOCIADOS AL PAGO POR SERVICIOS AMBIENTALES EN UNA COMUNIDAD MAYA DE CAMPECHE: CARACTERÍSTICAS Y ATRIBUTOS SOCIALES PARA SU IMPLEMENTACIÓN
}

\section{THE ARTIFICIAL NESTS ASSOCIATED WITH THE PAYMENT FOR ENVIRONMENTAL SERVICES IN A MAYAN COMMUNITY OF CAMPECHE: CHARACTERISTICS AND SOCIAL ATTRIBUTES FOR ITS IMPLEMENTATION}

\author{
Jimena ILLESCAS y SALVAdor MONTIEL*
}

\begin{abstract}
Laboratorio de Ecología y Conservación de la Biodiversidad (LAECBIO), Departamento de Ecología Humana. Centro de Investigación y de Estudios Avanzados del Instituto Politécnico Nacional (CINVESTAV-IPN), Unidad Mérida. Km 6 antigua carretera a Progreso, Mérida, 97310 Yucatán, México.<jimeniux@gmail.com>

*Autor para correspondencia: <montiels@cinvestav.mx>
\end{abstract}

Recibido: 22/05/2017; aceptado: 18/10/2017; publicado en línea: 16/03/2018

Editor responsable: Octavio Rojas Soto

Illescas, J. y Montiel, S. (2018) Los nidos artificiales asociados al pago por servicios ambientales en una comunidad maya de Campeche: características y atributos sociales para su implementación. Acta Zoológica Mexicana (n.s), 34(1), 1-4.

RESUMEN. Los nidos artificiales constituyen una estrategia de conservación para las aves silvestres en las áreas comunitarias vinculadas al Pago por Servicios Ambientales (PES) en México. Sin embargo, esta estrategia de nido artificial carece de directrices técnicas oficiales para su implementación, y exige en consecuencia la capacidad de los actores locales para llevarla a cabo en contextos socioambientales específicos. Aquí, describimos los nidos artificiales vinculados a PES, resaltando las capacidades locales y las percepciones sociales que subyacen a esta estrategia de conservación en una comunidad maya de Campeche, México. Con base en observaciones de campo y entrevistas semiestructuradas aplicadas a personas locales (campesinos-pescadores), que participan en PSA, encontramos que los nidos artificiales registrados $(\mathrm{N}=42)$ fueron considerados solo por el $52 \%$ de los entrevistados como "sitios de anidación" para al menos 25 especies de aves regionales. Nuestros resultados muestran que en la actualidad las características, la ubicación y las especies objetivo asociadas a los nidos artificiales dependen solo de las habilidades y capacidades de los interesados locales.

En México, el Pago por Servicios Ambientales (PSA) constituye un programa social (implementado desde 2003) que forma parte de la estrategia nacional para contrarrestar el cambio climático y la pérdida de la biodiversidad (CO-
Illescas, J., \& Montiel, S. (2018) The artificial nests associated with the payment for environmental services in a Mayan community of Campeche: characteristics and social attributes for its implementation. Acta Zoológica Mexicana (n.s), 34(1), 1-4.

ABSTRACT. Artificial nests constitute a conservation strategy for wild birds in community areas linked to Payment for Environmental Services (PES) in Mexico. However, this artificial nest strategy lacks of official technical guidelines for its implementation, demanding consequently capabilities of local stakeholders to carry out it in specific socio-environmental contexts. Here, we describe the artificial nests linked to PES, highligthing local capabilities and social perceptions underlying this conservation strategy in a Mayan community of Campeche, Mexico. Based on field observations and semi-structured interviews applied to local people (peasant-fishermen) partipating in PES, we found that the registered artificial nests $(\mathrm{N}=42)$ were considered only by $52 \%$ of the interviewees as "nesting sites" for at least 25 regional bird species. Our results show that nowdays the features, placement and target species associated to the artificial nests depend only of skills and capabilities of local stakeholders.

NAFOR 2012, Pervochtchikova, 2014). La elaboración y colocación de nidos artificiales para aves silvestres es una de las actividades elegibles por parte de los beneficiarios del PSA comprometidos con el salvaguardo de áreas 
orientadas a la conservación de la biodiversidad. Oficialmente, solo se refiere a los nidos artificiales asociados al PSA como "una actividad que contribuye a la protección y el manejo de los hábitats o especies, esencial para la subsistencia de poblaciones de fauna silvestre, migratoria o residente, sin que su hábitat se vea afectado por factores causados por el hombre" (DOF, 2016). Así, se carece de lineamientos técnicos para la implementación de estos nidos artificiales, omitiéndose aspectos centrales como el reconocimiento de la avifauna de interés, los recursos disponibles y las condiciones espacio-temporales requeridas para la adecuada operación de esta actividad, entre otros. Bajo estas condiciones, la operación de la actividad principalmente en áreas ejidales de nuestro país (ca. 51\% del territorio nacional; RAN, 2012), parece depender solo de iniciativas, capacidades y recursos locales de quienes solicitan la implementación de nidos artificiales como parte del PSA en su entorno ejidal. Empezar a documentar lo anterior es fundamental para evaluar la factibilidad de los nidos artificiales como una estrategia optativa del PSA para la conservación de la biodiversidad de aves en nuestro país.

En comunidades rurales de la Península de Yucatán, es posible asumir que la implementación de los nidos artificiales está ligada al conocimiento tradicional vinculado a su vez, con la estrategia de uso múltiple de la naturaleza que ha caracterizado a la cultura maya peninsular (Barrera-Bassols \& Toledo, 2005; Porter-Bolland et al., 2006). Se espera que las comunidades mayas que optan por la implementación de nidos artificiales, retomen usos y costumbres locales para operar inicialmente esta actividad en aquellas áreas ejidales comprometidas con la conservación de la biodiversidad como parte del PSA. Para explorar lo anterior, describimos los tamaños, materiales y sitios de colocación de nidos artificiales implementados por pobladores de una comunidad maya del oeste de Campeche, como un proxy de las capacidades locales asociadas a esta actividad del PSA.

El estudio se realizó en Tankuché Campeche (1953' $24^{\prime \prime} \mathrm{N}, 89^{\circ} 52^{\prime} 48^{\prime \prime} \mathrm{O}$ ), una comunidad maya de 1006 habitantes (INEGI 2010) ubicada al occidente de la Península de Yucatán, aledaña a la Reserva de la Biósfera Los Petenes (RBLP). Esta reserva abarca un humedal de importancia internacional para la conservación de las aves (sitio RAMSAR), caracterizado por una colección de fragmentos forestales o petenes (constituidos principalmente por vegetación arbórea de selva-manglar desarrollada alrededor de afluentes de agua dulce o cenotes; Rico-Gray 1982) inmersos en una matriz inundable de pastizal, ma- torral xerófilo y suelos desnudos o "blanquizales" (Flores \& Espejel, 1994; Montiel et al., 2008; Acosta-Lugo et al., 2010). Las principales actividades productivas de los pobladores de Tankuché son la agricultura de temporal y la pesca ribereña, siendo la elaboración de artesanías, la apicultura, la cacería de subsistencia, la extracción de palma de huano (Sabal yapa) y el trabajo asalariado (e.g., empleados en la industria del transporte y la construcción), actividades secundarias para hombres y mujeres de la comunidad (Méndez-Cabrera \& Montiel, 2007).

Tankuché está conformado legalmente como un ejido de 290 miembros, siendo $57 \%$ de ellos beneficiarios del PSA al momento del estudio. Desde el año 2009, Tankuché obtuvo un primer periodo quinquenal del PSA por conservación de la biodiversidad para un área incluida dentro del polígono de la RBLP. Para un segundo periodo (2013-2017), el PSA otorgado fue para un área ejidal (809 ha) ubicada fuera de la RBLP. En ambos periodos, el PSA incluyó la apertura y mantenimiento de una brecha cortafuego (perimetral al área del PSA) y solo para el segundo periodo, dicho programa incluyó también la elaboración y colocación de nidos artificiales para aves silvestres. Por indicaciones del técnico forestal, los participantes del PSA (165 ejidatarios) implementaron en esta actividad (durante el año 2014) cerca de 300 nidos artificiales a lo largo de la brecha cortafuego.

En agosto-septiembre 2015 (mediados de la temporada lluviosa regional), se realizaron 12 recorridos de campo a lo largo de la brecha cortafuego para registrar y caracterizar todos los nidos artificiales potencialmente detectables y que fueron colocados a principios del segundo quinquenio del PSA en la comunidad de estudio. Para cada nido se registró su tamaño (dos diámetros), material de elaboración, altura sobre el suelo y lugar de colocación (especie arbórea/arbustiva), obteniéndose un registro fotográfico en cada caso. Asimismo, con base en la lista de beneficiarios del PSA proporcionada por el comisario ejidal, un total de 86 beneficiarios (52\% del total) fueron seleccionados de forma aleatoria para la aplicación de una entrevista semiestructurada sobre los nidos artificiales implementados a nivel local. Un taller participativo con los beneficiarios del PSA, realizado al final de este estudio, permitió una validación colectiva de la información obtenida durante los recorridos de campo y las entrevistas.

Se encontró que 42 nidos ( $70 \%$ evidentes al observador y el resto detectables solo entre la vegetación) fueron accesibles para ser registrados in situ a lo largo de la brecha cortafuego. En promedio ( $\pm \mathrm{EE}$ ), esos nidos tuvieron un diámetro de $31.4 \mathrm{~cm}( \pm 1.2)$ y fueron colocados a una 
altura de $2.8 \mathrm{~m}( \pm 0.1)$ sobre especies leñosas como el jabín (Piscidia piscipula), el chucúm (Harvardia albicans), el kitinché (Caesalpinia gaumeri) y el calabazo (Crescentia cujete), las cuales representaron conjuntamente los lugares de colocación para $76 \%$ de los nidos. Los nidos registrados fueron elaborados localmente a partir de materiales rústicos (e.g., hojas, cortezas y ramas) derivados de especies vegetales encontradas en las inmediaciones del ejido: $74 \%$ pasto y zacate (Typha dominguensis, Cladium jamaicensis, Phragmites australis), 12\% palma de coco (Cocos nucifera), 7.1\% jipi (Carludovica palmata) y en menor medida $(<7 \%$ ) guano (Sabal yapa), plátano (Musa paradisiaca) y especies no identificadas $(2.3 \%$ en cada caso, respectivamente). A partir de su elaboración local y materiales rústicos es posible considerar a los nidos registrados como "nidos artesanales".

La opinión de los entrevistados sobre la utilidad de los nidos estuvo dividida. Con base en evidencia anecdótica, solo el $52 \%$ de ellos consideró que los nidos artificiales pueden servir como "sitios de anidamiento" para varias aves de su entorno. El resto de los entrevistados (48\%) consideró que tales estructuras, a pesar de elaborarse con insumos provenientes de la vegetación local, no son de utilidad para las aves ya que éstas hacen sus propios nidos. Cabe mencionar que durante los recorridos de campo no se encontró evidencia de uso (e.g., presencia de plumas, huevos o excretas) de dichos nidos artesanales, por parte de aves o algún otro vertebrado terrestre.

Asimismo, los entrevistados manifestaron que al no contar con guías o asesoría técnica para la implementación de nidos artificiales, las habilidades manuales y conocimientos tradicionales asociados a ciertas actividades productivas cotidianas (e.g., elaboración de artesanias, techos de palma, hamacas y accesorios de pesca) les permiten contar con insumos básicos para poder implementar esta actividad del PSA en su ejido. Además, se encontró que solo con base en información anecdótica y observaciones cotidianas, los participantes del taller comunitario (realizado en marzo 2016) reconocieron un total de 25 especies de aves como usuarias potenciales de los nidos artificiales colocados como parte del PSA en el ejido. Consideraron que por su tamaño, forma y material, esos nidos podrían ser sitios útiles principalmente para la paloma de monte (Zenaida asiatica) y la chachalaca (Ortalis vetula). Incluso, mencionaron que el pavo de monte (Meleagris ocellata) (a pesar de no ser candidato para ocupar este tipo de nidos; González et al., 1998), podrían encontrar en los nidos artificiales espacios alternativos de refugio en la vegetación arbórea.

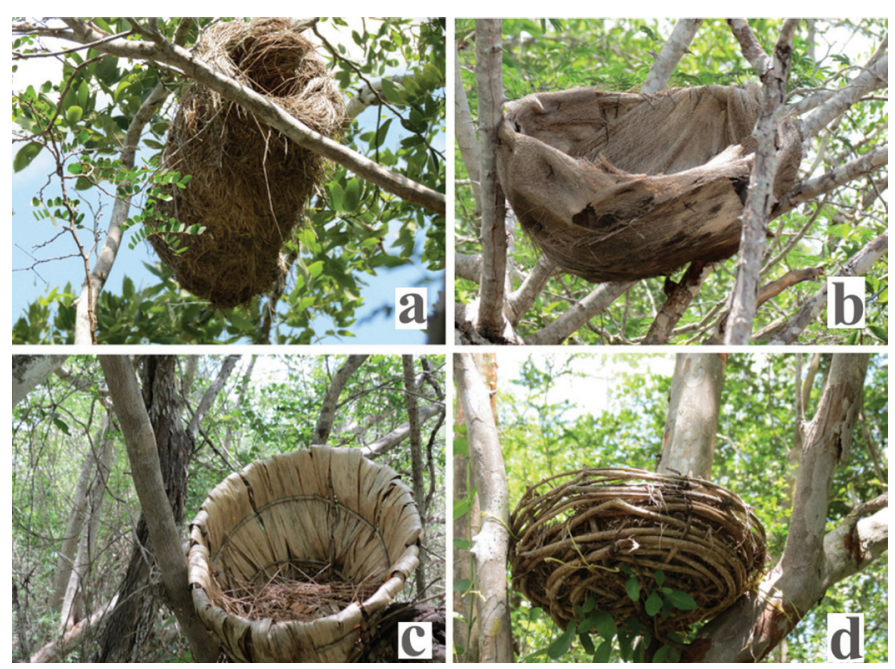

Figura 1. Muestras de nidos artificiales colocados como parte del Programa de Pago por Servicios Ambientales 2015 en Tankuché, Campeche, México. Nótese el entramado de materiales vegetales en cada caso: a) graminoides (pastos) arreglados a manera de saco colgado en Piscidia piscipula, b) tallos de palma de coco arreglados a manera de cesto sobre Harvardia albicans, c) hojas de palma de guano arregladas a manera de cesto colocado sobre Caesalpinia gaumeri, d) lianas o bejucos arreglados a manera de cesto sobre un árbol (no identificado).

Si bien la implementación de nidos artificiales podría constituir una alternativa importante para la conservación de ciertas especies de aves (e.g., uso de cajas nido para falcónidos; Katzner et al., 2005), esta estrategia exige un soporte técnico claro para llevarla al cabo, principalmente utilizando diseños probados para grupos de aves específicos en los ambientes de interés (Fig. 1) (Muñoz-Pedreros et al., 1996; Klein et al., 2007). Al carecerse de lo anterior, la implementación de nidos artificiales en comunidades mayas como Tankuché dependerá solo de iniciativas, capacidades y recursos locales de quienes soliciten un PSA para alguna parte de su ejido. Estas capacidades locales deben tomarse en cuenta por la autoridad ambiental para el diseño e implementación de acciones comunitarias orientadas a la conservación de la biodiversidad.

AGRADECIMIENTOS. Agradecemos la confianza otorgada por los pobladores de Tankuché para permitirnos conocer y documentar parte de su experiencia del PSA. Asimismo, agradecemos los fondos, facilidades y apoyo logístico brindados por el CINVESTAV-IPN Unidad Mérida para la realización del presente estudio. Julio Casanova y Armando Rojas brindaron un valioso apoyo en campo. Este estudio formó parte de la tesis de maestría del primer autor (JI), quién contó con una beca (\#397501) otorgada por el Consejo Nacional de Ciencia y Tecnología de México (CONACYT). 


\section{LITERATURA CITADA}

Acosta-Lugo, E., Alonzo-Parra, D., Andrade-Hernández, M., Castillo-Tzab, D., Chablé-Santos, J., Durán, R. \& GonzálezIturbe, J. (2010). Plan de Conservación de la Eco-región Petenes-Celestún-Palmar. Pronatura. Mérida, Yucatán, 177 pp.

Barrera-Bassols, N. \& Toledo, V. M. (2005) Ethnoecology of the Yucatec Maya: symbolism, knowledge and management of natural resources. Journal of Latin American Geography, 4, 9-41.

CONAFOR (Comisión Nacional Forestal). (2012) Guía de Mejores Prácticas de Manejo (GMPM) 2012, correspondiente a las áreas de pago diferenciado 3, 4, 5 y 6. Comisión Nacional Forestal y Secretaría de Medio Ambiente y Recursos Naturales. México.

DOF (Diario Oficial de la Federación). (2016) Reglas de Operación del Programa Nacional Forestal 2016. México, Distrito Federal, 31 de diciembre de 2015.

Flores, J. S. \& Espejel, I. (1994) Tipos de vegetación de la Península de Yucatán. Etnoflora Yucatanense, Fascículo 3. Facultad de Medicina Veterinaria y Zootecnia, Universidad Autónoma de Yucatán, Mérida, México, 135 pp.

González, M. J., Quigley, H. B. \& Taylor, C. I. (1998). Habitat use and reproductive ecology of the Ocellated Turkey in Tikal National Park, Guatemala. Wilson Bulletin, 110, 505-510.

INEGI (Instituto Nacional de Geografía e Informática). (2010) Censo de población y vivienda 2010. Disponible en: http://www.inegi. org.mx/sistemas/consulta_resultados/iter2010.aspx (fecha de consulta: marzo 2015).

Katzner, T., Obertson S., Robertson B., Klucsarits, J., Mccarty K. \& Bildstein K. L. (2005) Results from a long-term nest-box program for American Kestrels: implications for improved population monitoring and conservation. Journal of Field Ornithology,
76, 217-226.

Klein, Á., Nagy, T., Csörgő T. \& Mátics, R. (2007) Exterior nestboxes may negatively affect Barn Owl Tyto alba survival: an ecological trap. Bird Conservation International, 17, 273-281.

Méndez-Cabrera F. \& Montiel, S. (2007). Diagnóstico preliminar de los recursos silvestres usados por los Mayas de dos comunidades costeras de Campeche, México. Universidad y Ciencia, 23, 127139.

Montiel S., León, P. \& Estrada, A. (2008) Riqueza y diversidad de quirópteros en hábitats-isla en una región naturalmente fragmentada de Mesoamérica. Pp. 373-392. En: J. C. Sáenz \& Harvey C. (eds). Evaluación y conservación de la biodiversidad de paisajes fragmentados de Mesoamérica. Editorial InBIO, Costa Rica.

Muñoz-Pedreros, A., Gantz, A. \& Saavedra, M. (1996) Nidos artificiales en plantaciones de Pinus radiata en el sur de Chile: ¿una herramienta para mitigar impactos ambientales negativos? Revista Chilena de Historia Natural, 69, 393-400.

Perevochtchikova, M. (2014) Programa de Pago por Servicios Ambientales en México: hacia nuevos esquemas de evaluación. Pp. 581-609. En: M. Perevochtchikova (coord.). Pago por Servicios Ambientales en México. Un acercamiento para su estudio, coordinadora México. COLMEX, México.

Porter-Bolland, L., Drew, A. \& Vergara-Tenorio, C. (2006) Analysis of a Natural Resources Management System in the Calakmul Biosphere Reserve. Landscape and Urban Planning, 74, 223-24.

RAN (Registro Agrario Nacional). (2012) Disponible en: http://www. gob.mx/ran/prensa/ejidos-y-comunidades-fundamentales-para-eldesarrollo-del-pais-ran (fecha de consulta: abril 2017).

Rico-Gray, V. (1982) Estudio de la vegetación de la zona costera inundable del noroeste del Estado de Campeche, México, Los Petenes. Biotica, 7, 171-190. 